\title{
A Realidade Aumentada na prática de exercícios físicos
}

Luana Bittencourt Saraiva Programadora Visual na Assessoria de Comunicação da Universidade Federal do Amazonas (Ascom/Ufam). Mestre no Programa da Pós-graduação em Design na Universidade Federal do Amazonas (PPGD-UFAM). Especialista em Design, Comunicação e Multimídia pela Faculdade Fucapi. Graduada em Design pela Universidade Federal do Amazonas (UFAM) e em Administração pela Universidade do Estado do Amazonas (UEA).

luana@super.ufam.edu.br ORCID 0000-0001-6117-6980

Bruno Raphael de Carvalho Santos Mestre em Design pela Universidade Federal do Amazonas (PPGD-UFAM) com foco em Gestão do Design para Micro e Pequenas Empresas. Formou-se em design pela Faculdade Martha Falcão (FMF) com especialização em Design, Comunicação e Multimídia pela Fundação Centro de Análise, Pesquisa e Inovação (FUCAPI) em 2014. Sua área de interesse atual é gestão de negócios, tecnologia, educação e design. brunoraphael@ufam.edu.br ORCID 0000-0002-4223-0176
Resumo Diante da pandemia do novo coronavírus, importam iniciativas capazes de adaptar-se ao isolamento social e às tecnologias de operação remota. Entre as tecnologias emergentes, a Realidade Aumentada (RA) permite interação entre o mundo real e virtual, auxiliando inclusive, pessoas a terem uma vida mais saudável. 0 artigo investiga como a RA tem sido utilizada para orientar pessoas na realização de atividade físicas e se dá pela prospecção em bases de dados internacionais para visualizar a abordagem da temática. Os resultados mostraram dois grupos de aplicações em RA: a gamificação, protagonizada pelo jogo Pokémon Go; e a reabilitação, com aplicações para recuperação de pessoas com perda muscular. Constata-se predomínio de publicações originadas nos Estados Unidos e Reino Unido, conforme mecanismo de busca e string definida para realização da pesquisa de prospecção nas coleções de periódicos. As informações coletadas auxiliarão na definição de funcionalidades que poderão ser utilizadas em aplicações em RA.

Palavras-Chave Realidade Aumentada, Atividades Físicas, Revisão de Literatura. 


\section{+ TECNOLOGIA}

Alef Vernon De Oliveira Santos Graduado em Design pela Universidade Federal do Amazonas, com foco em User Interface Design e User Experience Design, atuante como Product Designer em Plataformas Digitais e Ilustrador 2D. Mestrando do Programa de Pós-Graduação em Design da Universidade Federal do Amazonas.

alef@super.ufam.edu.br

ORCID 0000-0002-1261-9623

Eduardo Jorge Lira Antunes Da Silva Licenciado em Computação pela UEA; Mestrando do Programa de Pós-Graduação em Design da UFAM; Especialista em Design Digital; Técnico em Computação Gráfica.

eduardo@super.ufam.edu.br

ORCID 0000-0002-2233-703X

Lúcio Vasconcellos Dias Designer de produto da Planta Projetos e Instalações de Móveis LTDA-ME. Mestrando do Programa de Pós-Graduação em Design da Universidade Federal do Amazonas. Pós Graduando em Design de Interiores pelo Instituto de Pós Graduação e Graduação - IPOG. Graduado em Design pela Faculdade Martha Falcão. lucio@super.ufam.edu.br ORCID 0000-0003-2585-4267

\section{Augmented reality in physical exercise}

Abstract Faced with the new coronavirus pandemic, initiatives capable of adapting to social isolation and remote operation technologies are relevant. Among the emerging technologies, Augmented Reality (AR) allows interaction between the real and virtual world, even helping people to have a healthier life. The article investigates how AR has been used to guide people in performing physical activity and takes place through prospecting in international databases to visualize the approach to the theme. The results showed two groups of applications in AR: gamification, played by the Pokémon Go game; and rehabilitation, with applications for the recovery of people with muscle loss. There is a predominance of publications originating in the United States and the United Kingdom, according to the search engine and defined string for conducting the prospecting research in journal collections. The information collected will help to define functionalities that can be used in RA applications.

Keywords Augmented Reality, Physical Activities, Literature Review.

\section{Realidad aumentada en la práctica de ejercicios físicos}

Resumen Ante la nueva pandemia de coronavirus, son importantes las iniciativas capaces de adaptarse al aislamiento social y las tecnologías de operación remota. Entre las tecnologías emergentes, la Realidad Aumentada (AR) permite la interacción entre el mundo real y virtual, ayudando incluso a las personas a tener una vida más saludable. El artículo investiga cómo se ha utilizado la RA para orientar a las personas en la realización de actividad física y se realiza mediante la prospección en bases de datos internacionales para visualizar el abordaje del tema. Los resultados mostraron dos grupos de aplicaciones en AR: gamificación, jugado por el juego Pokémon Go; y rehabilitación, con aplicaciones para la recuperación de personas con pérdida muscular. Predominan las publicaciones con origen en Estados Unidos y Reino Unido, según el buscador y la cadena definida para realizar la investigación de prospección en las colecciones de revistas. La información recopilada ayudará a definir funcionalidades que se pueden utilizar en aplicaciones de RA.

Palabras clave Realidad Aumentada, Actividades Físicas, Revisión de Literatura. 
Claudete Barbosa Ruschival Doutora em Engenharia de Produção pela Universidade Federal de Santa Catarina (2012) onde também fez mestrado em Engenharia de Produção (2004). Formou-se em Desenho Industrial pela Universidade Federal do Amazonas (UFAM) em 1996. Especialista em Design de Embalagens bolsista do Posgrado Mercosul Design Program, uma cooperação italiana, espanhola e do Mercosul (2001) e em Publicidade e Marketing pela Universidade Federal do Amazonas (1998). Atua como professora associada do curso de Design e do Programa de Pós-Graduação em Design. Tem vasta experiência na área de Design Industrial, com ênfase em Processos de Desenvolvimento de Produto e Gestão de Design.

claudete@super.ufam.edu.br ORCID 0000-0001-5686-3209

Nelson Kuwahara Professor Associado da UFAM. Coordenador do Laboratório TRANSPORTAR, Departamento de Design e Expressão Gráfica, Faculdade de Tecnologia, Universidade Federal do Amazonas - UFAM. Graduado em Engenharia Mecânica pela Universidade Federal do Pará. Mestrado em Planejamento de Sistemas Energéticos pela Faculdade de Engenharia Mecânica, Universidade Estadual de Campinas. Doutorado em Engenharia de Transportes, PET - COPPE - Universidade Federal do Rio de Janeiro. Coordenador do Programa de Pós-Graduação em Design - PPGD, e Professor dos Programas de Pós-Graduação em Engenharia de Produção (PPGEP) e Propriedade Intelectual e Transferência de Tecnologia para a Inovação (PPG-PROFNIT) da UFAM.

nelson@super.ufam.edu.br ORCID 0000-0002-4572-9415

\section{Introdução}

A prática regular de exercícios físicos é fator de prevenção e controle de doenças não transmissíveis, como doenças cardiovasculares, diabetes tipo 2, câncer de mama e cólon. Além disso, a atividade física também traz benefícios para a saúde mental, retarda o aparecimento da demência e pode contribuir para a manutenção do peso saudável e do bem-estar geral (WHO, 2020). O sedentarismo, por sua vez, está associado a diabetes tipo 2, bem como doenças cardiovasculares, câncer e mortalidade por todas as causas, segundo a Organização Mundial da Saúde - OMS (2020), além de impactar no bem-estar e na qualidade de vida dos indivíduos.

Sabe-se que a inovação tecnológica e a transição para ocupações e recreação com pouca movimentação, além do uso crescente de transporte pessoal motorizado, são contribuintes para a mudança dos padrões de atividade física e para o aumento do comportamento sedentário em todo o mundo (WHO, 2020).

De acordo com WHO (2020), todos os adultos entre 18 e 64 anos devem praticar atividade física regular, realizando pelo menos 150 minutos/ semana de atividade física aeróbica de intensidade moderada; ou 75 minutos/semana de atividade física aeróbica de intensidade vigorosa; ou uma combinação de ambos, para benefícios substanciais à saúde. Essas recomendações ainda podem ser alcançadas mesmo em casa, sem equipamentos especiais e com espaço limitado.

Com a pandemia causada pelo novo coronavírus, a população mundial foi levada a se isolar socialmente, o que trouxe mudanças radicais para a rotina e os hábitos tanto pessoais quanto profissionais, afetando o estado físico e mental de todos. Essa nova problemática demanda iniciativas que ajudem as pessoas a se adaptarem a realidade atual, apontando novas formas de trabalho e de socialização, restritas hoje ao ambiente familiar e por meio das tecnologias de operação remota.

Diante de tais restrições, popularizou-se o uso de aplicativos voltados à prática de exercícios físicos, cujo fim é ajudar as pessoas a inovar seu estilo de vida, permitindo a execução de atividades em casa. A realidade virtual (RV) e a realidade aumentada (RA) são exemplos de tecnologias emergentes que proporcionam impactos positivos na prática de atividades físicas, que aprimoram a experiência e a imersão do usuário na interação entre o mundo real e o mundo virtual.

Dada a importância da prática de exercícios físicos para a saúde e bem-estar, aliada a existência de tecnologias emergentes que abrem amplas possibilidades de aplicação, inclusive para práticas físicas, o presente estudo tem como objetivo investigar o estado da arte da RA aplicada na vida fitness, para entender como esta tecnologia tem sido utilizada para orientar usuários nas atividades físicas existentes. Ainda, verificar o uso eficaz dessa tecnologia enquanto instrumento de melhoria da qualidade de vida pela diminuição do sedentarismo. Para atingir o objetivo do estudo, realizou-se uma prospecção e análise em bases de dados internacionais, a fim de visua- 
lizar como a temática vem sendo abordada no mundo e apontar elementos específicos e metodologias para serem aplicadas em pesquisa futura.

Conceitualmente, importa anotar que RA difere de realidade virtual. A RV corresponde a uma variação de virtual enviroments (VE) ou virtualização do ambiente, que insere um elemento virtual no ambiente real. Azuma (1997) afirma que as tecnologias VE imergem completamente o usuário dentro de um ambiente sintético, que, enquanto imerso, não pode ver o mundo real à sua volta. Em contraste, a RA permite ao usuário visualizar o mundo real, com objetos virtuais sobrepostos ou compostos com a realidade, complementando-a em vez de substituí-la completamente. Tais temáticas são amplamente abordadas por Milgram \& Kishino (1994), Jér $\square$ me Jetter et al. (2018). Ng, Ma, et al., (2019), Kamel Boulos et al. (2017).

\section{Metodologia}

O embasamento teórico e metodológico do presente estudo ancora-se no estado-da-arte sobre aplicação da RA em contextos ligados à prática de exercícios físicos. Também foram empregados métodos de pesquisa em uma área que possam indicar novos rumos para futuras investigações.

Tranfield, Denyer e Smart (2003), explicam que um trabalho de revisão se divide em três estágios: (1) Planejamento da Revisão, (2) Condução da Pesquisa e (3) Geração de Relatórios e Divulgação. A Figura 1 demonstra resumidamente e sistematicamente o processo metodológico de forma visual.

Figura 1 Aplicação do método proposto por Tranfield, Denyer e Smart (2003).

Fonte Os autores (2021)

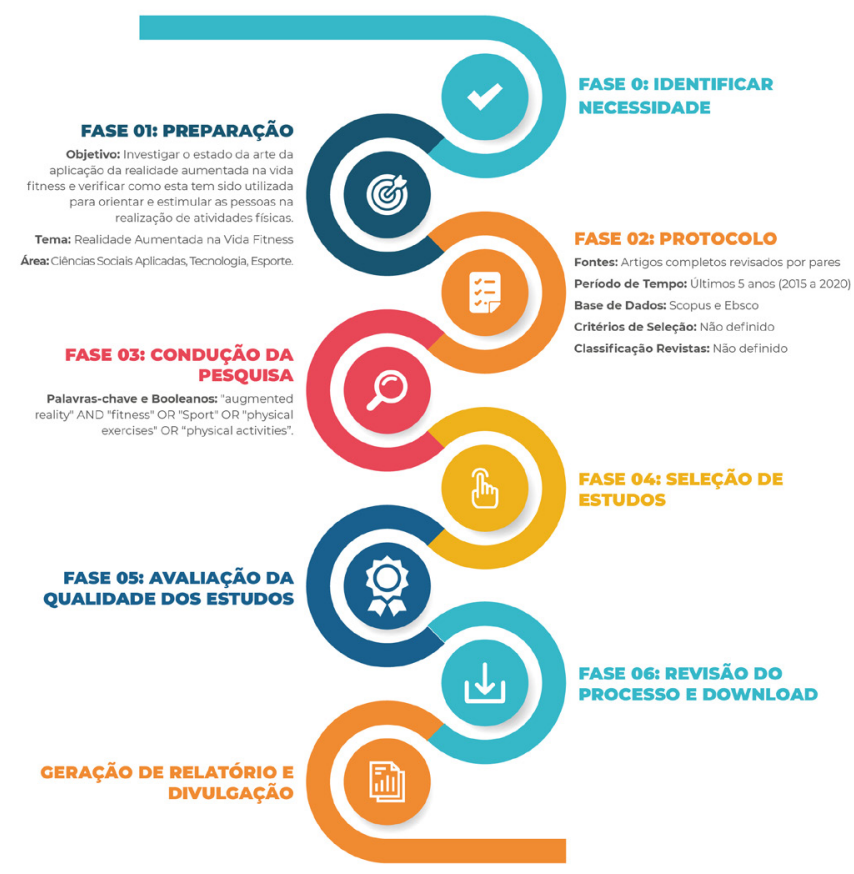


A partir da análise dos 13 artigos coerentes com os objetivos do estudo, tem-se como primeiro ponto de realce o retorno de muitos estudos associando o jogo de RA Pokémon Go à prática de exercícios físicos. Os potenciais benefícios para a saúde ao jogar Pokémon Go atraíram um interesse considerável de pesquisadores, com análises divulgando o impacto desse jogo sobre a atividade física e a saúde mental das pessoas.

No âmbito da base de dados Ebsco, dos nove artigos selecionados para análise, obteve-se o resultado: quatro deles tratavam de estudos de campo sobre o jogo Pokémon Go; três relacionados a reabilitação terapêutica; um com abordagem teórica das soluções de RV e RA para a saúde individual e pública, e; um tratava de uma revisão sistemática sobre a aplicação da RV e da RA para a melhoria do desempenho físico e psicológicos de uma população saudável.

Quanto à base de dados Scopus, foram quatro documentos de interesse da pesquisa. Um deles tratava do jogo Pokémon GO como incentivo à prática de atividade física. Outros três artigos analisavam os benefícios da RA quando aplicada as práticas físicas, sendo um direcionado a prática de Tai-chi, um segundo à prática aeróbica e de flexibilidade. Já o terceiro, apresentou revisão bibliográfica sobre a tecnologia aplicada à prática de exercícios físicos.

Cabe ainda ressaltar que as referências prospectadas na base Scopus também tiveram como fator de decisão a proeminência em número de citações na base, em que resultou 170 artigos na busca com a string "augmented reality" AND "fitness" OR "Sport" OR "physical exercises" OR "physical activities". A Figura 2 apresenta o grafo gerado pela ferramenta de análise bibliométrica VOSviewer, na qual tem-se a representação dos autores de maior destaque em número de citações entre todos os artigos prospectados.

Figura 2 Grafo com destaque dos artigos de maior número de citações entre os 170 prospectados na base Scopus.

Fonte Os autores (2021)

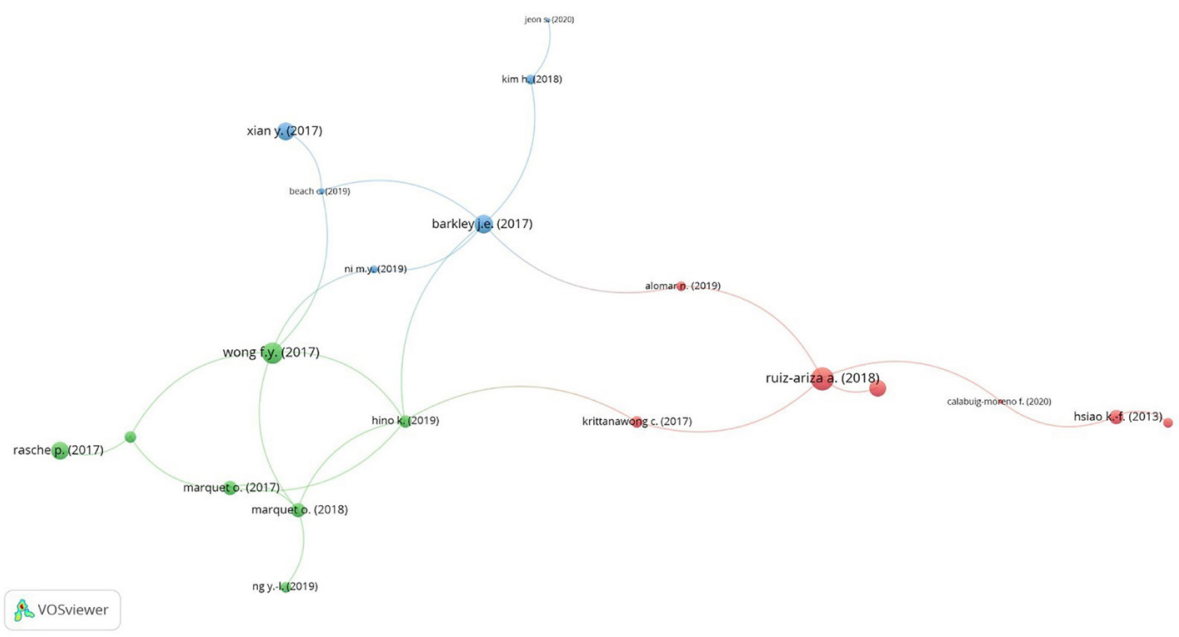

São 45 os países que apresentam produção dentro de tal temática, conforme o que consta no resultado da prospecção dos 170 artigos da base Scopus. Os dez primeiros países que mais publicam, de acordo com o nú- 
Figura 3 Subconjunto de 123 palavras-chaves que mais se destacam. Fonte Os autores (2021) mero de artigos prospectados, e com indicação do número de artigos são os seguintes: 32 Estados Unidos, 22 Reino Unido, 15 Espanha, 14 Taiwan, 14 Austrália, 12 Itália, 11 Coréia do Sul, 7 Alemanha, 7 Holanda, 7 Finlândia. Embora apresentando em menor número de publicações, China, Croácia, Arábia Saudita, Luxemburgo, são os países que demostram as produções mais recentes.

Considerando as 1590 palavras-chave totais presentes na prospecção da base Scopus, para os artigos que foram elegíveis pela busca com a string norteadora da pesquisa, o destaque das que apresentam pelo menos 3 ocorrências resulta em subconjunto de 123 palavras-chave que mais se repetem. Neste cenário as 4 palavras-chave que possuem maior número de ocorrências, e respectivo número de vezes que são citadas no conjunto dos artigos elegíveis são: RA (118 vezes), Realidade virtual ( 51 vezes), Humano (41 vezes) e Atividade física (37 vezes). A Figura 3 apresenta a plotagem das palavras que mais se destacam dentre as que são citadas pelos artigos, bem como suas conexões promovidas entre tais artigos.

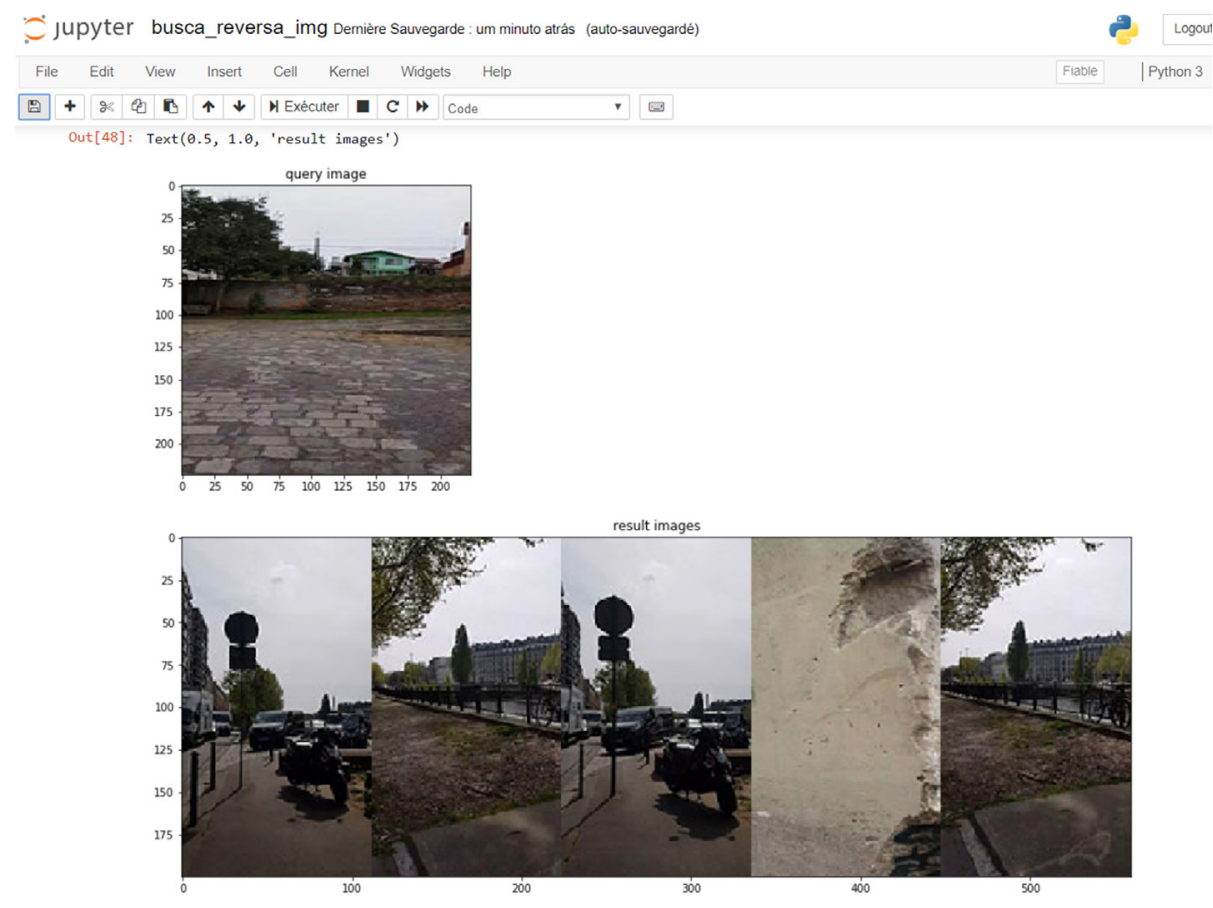

É possível verificar que a palavra Pokémon Go surge 16 vezes, embora representando ocorrência média, indica relativa proeminência dentre as palavras-chave, mas indica principalmente rede de conexão relevante entre as principais identificadas, conforme visualiza-se na Figura 4. Nota-se que a abordagem destas conexões tende para publicações mais recentes dentre todas as representadas no grafo gerado no VOSviewer. Assim, os artigos selecionados para proceder com as análises estão alinhados com tal cenário. 


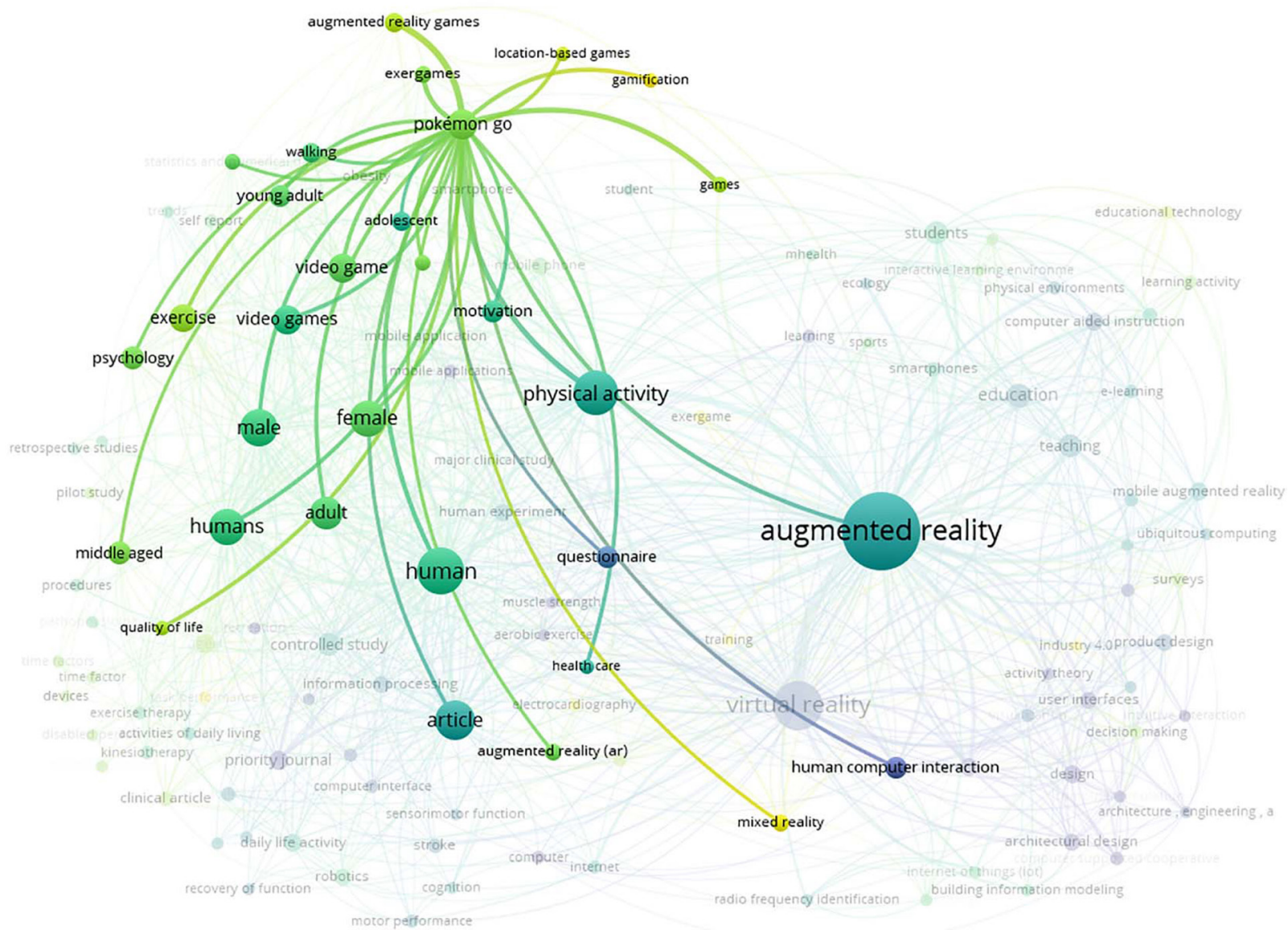

Figura 4 Rede de conexão gerada a partir da palavra-chave Pokémon Go.

Fonte Os autores (2021)

\section{Resultados}

Verificou-se que a abordagem da temática da RA aplicada na vida fitness pode ser categorizada em dois grupos: estudos relacionados ao jogo Pokémon Go, e; estudos relacionados a aplicação da RA para reabilitação terapêutica. Tais resultados são apresentados a seguir.

\section{Pokémon Go}

A popularidade do jogo de RA para smartphone Pokémon GO, suscitou múltiplas possibilidades quanto à sua utilização como mecanismo para aumentar os níveis de atividade física entre jovens adultos (MARQUET, ALBERICO e HIPP, 2018). o jogo, lançado em 2016, desenvolvido pela empresa Niantic, Inc. é um "exergame" social de RA, baseado em localização móvel. É de interesse para este estudo por motivar pessoas a se movimentarem, e talvez seja o exemplo mais popular de todos os tempos de um aplicativo de RA direcionado à saúde (BOULOS et al., 2017).

Devido ao jogo estar associado à prática de atividades físicas, verifica-se que Pokémon Go foi objeto de estudo em cinco artigos analisados nesta revisão, conforme mostrado no Quadro 01. 


\begin{tabular}{|c|c|c|c|c|c|}
\hline & Artigo & Autores & Foco do Estudo & Tipo de Pesquisa & Amostra \\
\hline 1 & $\begin{array}{l}\text { Effect of augmented } \\
\text { reality game Pokemon } \\
\text { GO on cognitive perfor- } \\
\text { mance and emotional } \\
\text { intelligence in adoles- } \\
\text { cent Young }\end{array}$ & $\begin{array}{l}\text { Ruiz-Ariza et } \\
\text { al (2018) }\end{array}$ & $\begin{array}{l}\text { Analisar o efeito do jogo no } \\
\text { desempenho cognitivo e } \\
\text { inteligência emocional }\end{array}$ & $\begin{array}{l}\text { Pesquisa de Campo por } 8 \\
\text { semanas }\end{array}$ & $\begin{array}{l}\text { Adolescentes espanhóis } \\
\text { de } 12 \text { a } 15 \text { anos. }\end{array}$ \\
\hline 2 & $\begin{array}{l}\text { A pilot investigation of } \\
\text { the physical and psy- } \\
\text { chological benefits of } \\
\text { playing Pokemon GO for } \\
\text { dog owners }\end{array}$ & $\begin{array}{l}\text { Kogan et al } \\
(2017)\end{array}$ & $\begin{array}{l}\text { Avaliar os comportamen- } \\
\text { tos de saúde e as percep- } \\
\text { ções de jogadores adultos, } \\
\text { donos de cães e jogadores } \\
\text { de Pokémon GO dos EUA. }\end{array}$ & $\begin{array}{l}\text { Pesquisa de Campo: Questio- } \\
\text { nário on-line }\end{array}$ & $\begin{array}{l}\text { Maiores de } 18 \text { anos, mo- } \\
\text { rador dos EUA, jogadores } \\
\text { de Pokémon Go e donos } \\
\text { de cachorro. Responden- } \\
\text { tes: } 18 \text { a } 29 \text { anos. }\end{array}$ \\
\hline 3 & $\begin{array}{l}\text { Influence of Pokémon } \\
\text { Go on physical activity } \\
\text { levels of university } \\
\text { players: a cross-sectio- } \\
\text { nal study }\end{array}$ & Wong (2017) & $\begin{array}{l}\text { Investigar o impacto do } \\
\text { Pokémon Go na atividade } \\
\text { física de estudantes em } \\
\text { universidade de Hong } \\
\text { Kong. }\end{array}$ & $\begin{array}{l}\text { Pesquisa de Campo: Questio- } \\
\text { nário on-line }\end{array}$ & 644 universitários \\
\hline 4 & $\begin{array}{l}\text { Pokémon G0 and its } \\
\text { Influence on Spanish } \\
\text { Facebook Users }\end{array}$ & $\begin{array}{l}\text { Escaravajal- } \\
\text {-Rodríguez } \\
\text { (2018) }\end{array}$ & $\begin{array}{l}\text { Estudar a influência do } \\
\text { Pokémon Go nos usuários } \\
\text { espanhóis do Facebook em } \\
\text { termos de atividade física, } \\
\text { relações sociais, orienta- } \\
\text { ção espacial e lugares. }\end{array}$ & $\begin{array}{l}\text { Pesquisa de Campo: Questio- } \\
\text { nário on-line }\end{array}$ & $\begin{array}{l}714 \text { indivíduos residentes } \\
\text { na Espanha. Faixa etária } \\
\text { de } 11 \text { a } 56 \text { anos.Apenas } \\
\text { usuários presentes em } 79 \\
\text { grupos de Pokémon no } \\
\text { Facebook }\end{array}$ \\
\hline 5 & $\begin{array}{l}\text { Pokemon G0 and phy- } \\
\text { sical activity among } \\
\text { college students. A } \\
\text { study using Ecological } \\
\text { Momentary Assessment }\end{array}$ & $\begin{array}{l}\text { Marquet, Al- } \\
\text { berico e Hipp } \\
(2018)\end{array}$ & $\begin{array}{l}\text { Analisar a relação entre } \\
\text { atividade física e com- } \\
\text { portamentos de jogo, em } \\
\text { jogadores de Pokémon GO } \\
\text { e não jogadores. }\end{array}$ & $\begin{array}{l}\text { Pesquisa de Campo: pesquisa } \\
\text { on-line pré e pós instalação da } \\
\text { ferramenta de Avaliação Mo- } \\
\text { mentânea Ecológica (EMA) e } \\
\text { contador de passos em smar- } \\
\text { tphones. Duração: } 7 \text { dias. }\end{array}$ & 74 universitários dos EUA \\
\hline 6 & $\begin{array}{l}\text { Pokemon GO and Phy- } \\
\text { sical Activity in Asia: } \\
\text { Multilevel Study }\end{array}$ & $\begin{array}{l}\text { MA et al } \\
(2018)\end{array}$ & $\begin{array}{l}\text { Estudar a relação entre o } \\
\text { uso de Pokémon G0 e a } \\
\text { atividade física dos joga- } \\
\text { dores e a variação com } \\
\text { diferentes níveis de ativi- } \\
\text { dade física. }\end{array}$ & $\begin{array}{l}\text { Pesquisa de campo: Medição } \\
\text { das distâncias médias diárias } \\
\text { de caminhada e corrida ao lon- } \\
\text { go de } 35 \text { dias - } 14 \text { dias antes } \\
\text { a } 21 \text { dias após a instalação } \\
\text { do jogo. }\end{array}$ & $\begin{array}{l}214 \text { pessoas entre } 13 \text { e } \\
65 \text { anos em Hong Kong. }\end{array}$ \\
\hline
\end{tabular}

Quadro 1 Estudos do jogo Pokémon Go associado à prática de atividades físicas

Fonte Os autores (2021)
A pesquisa de Ruiz-Ariza et al (2018), indica que os dados obtidos na pesquisa não mostraram mudanças significativas em jogadores adolescentes de Pokémon GO em relação ao bem-estar, autocontrole e emocionalidade.

A pesquisa de Kogan et al (2017), por sua vez, traz informações relacionadas à saúde e à percepção de pessoas adultas, donas de cães e jogadoras de Pokémon GO dos Estados Unidos. Declararam se sentirem menos ansiosos em relação a três situações geradoras 
de ansiedade: sair de casa, conversar com estranhos e ir a novos lugares.

No estudo de Wong (2017) os pesquisadores concluíram que, apesar de não ter sido identificado um impacto substancial nos níveis de atividade física dos usuários, Pokémon Go pode mesmo assim motivar jogadores, antes sedentários, a se tornarem mais ativos fisicamente. Logo, o jogo foi usado como ponto de partida para essas pessoas iniciarem um estilo de vida ativo.

O artigo de Escaravajal-Rodríguez (2018) mostra que houve aumento da atividade física dos jogadores, conhecimento de novas pessoas, ruas e pontos de interesse em sua cidade, alguns ainda alegaram terem aumentado suas saídas para a natureza. Assim, o estudo concluiu que Pokémon GO fomenta a atividade física, as relações sociais e a descoberta da cidade e da natureza. Os autores afirmam que a gamificação é um poderoso instrumento complementar de intervenção em programas de atividade física, além de ser capaz de promover o relacionamento social entre pessoas que compartilham da mesma atividade.

O estudo de Marquet, Alberico e Hipp (2018), apresenta que o acúmulo de três ou mais episódios de jogo ativo por dia foi associado ao aumento de 1.526 passos diários. Sob circunstâncias certas, como a hora do dia em que o jogo é utilizado, ou onde o jogo ocorre, Pokemon GO pode se tornar uma ferramenta útil para a promoção da saúde entre os jovens adultos.

Finalmente, Ma et al. (2018) conclui que jogos com RA tem grande potencial no combate ao sedentarismo, podendo ser utilizado como ferramenta global de intervenção pela saúde pública.

\section{Reabilitação Terapêutica}

Calabuig-Moreno et al. (2020) afirmam que a tecnologia tem sido utilizada de modo crescente na sociedade, o que levou os formuladores das políticas educacionais a estimular a adesão da tecnologia na sala de aula como incentivador da aprendizagem, bem como a aprendizagem de novas práticas físicas de reabilitação, seja preventiva ou para recuperação de habilidade motora.

A RA é uma tecnologia emergente que apresenta crescente número de pesquisas sobre exercícios em casos de reabilitação, mostrando que o seu uso pode ser aplicado para o aprendizado de práticas esportivas, como forma de viabilizar o exercício físico para promover qualidade de vida.

A organização e análise dos estudos encontrados na base de busca Scopus, mostraram como a RA pode ser utilizada no aprendizado de exercícios físicos de reabilitação. Os dados gerais dos artigos são apresentados no Quadro 2, a seguir. 
Quadro 2 Artigos da base Scopus sobre RA aplicada a exercícios físicos de reabilitação.

Fonte Os autores (2021)

\begin{tabular}{|c|c|c|c|c|c|}
\hline & Artigo & Autores & Foco do Estudo & $\begin{array}{l}\text { Tipo de Pes- } \\
\text { quisa }\end{array}$ & Público \\
\hline 1 & $\begin{array}{l}\text { The emergence of te- } \\
\text { chnology in physical } \\
\text { education: a general } \\
\text { bibliometric analysis } \\
\text { with a focus on vir- } \\
\text { tual and augmented } \\
\text { reality }\end{array}$ & $\begin{array}{l}\text { Calabuig- } \\
\text {-Moreno } \\
\text { et al. } \\
(2020)\end{array}$ & $\begin{array}{l}\text { Análise bibliomé- } \\
\text { trica dos artigos } \\
\text { publicados na Web } \\
\text { of Science (WoS) } \\
\text { sobre tecnologia em } \\
\text { Educação Física e } \\
\text { análise dos artigos } \\
\text { da pesquisa publica- } \\
\text { dos em RA ou RV em } \\
\text { Educação Física. }\end{array}$ & $\begin{array}{l}\text { Análise biblio- } \\
\text { métrica }\end{array}$ & $\begin{array}{l}\text { Artigos } \\
\text { publicados } \\
\text { na Web of } \\
\text { Science } \\
\text { (WoS) } \\
\text { sobre RA } \\
\text { ou RV na } \\
\text { Educação } \\
\text { Física. }\end{array}$ \\
\hline 2 & $\begin{array}{l}\text { Effects of Augmen- } \\
\text { ted-Reality-Based } \\
\text { Exercise on muscle } \\
\text { parameters, physical } \\
\text { performance, and } \\
\text { exercise self-efficacy } \\
\text { for older adults }\end{array}$ & $\begin{array}{l}\text { Jeon } \\
\text { \& Kim } \\
(2020))\end{array}$ & $\begin{array}{l}\text { Determinar a apli- } \\
\text { cabilidade de um } \\
\text { programa de exer- } \\
\text { cícios de prevenção } \\
\text { da redução muscular } \\
\text { baseado em RA. }\end{array}$ & $\begin{array}{l}\text { Pesquisa } \\
\text { aplicada ex- } \\
\text { perimental: } \\
30 \text { minutos de } \\
\text { exercícios, } 5 \\
\text { vezes por se- } \\
\text { mana, durante } \\
12 \text { semanas }\end{array}$ & $\begin{array}{l}27 \text { mulhe- } \\
\text { res idosas } \\
\text { coreanas } \\
\text { com mais } \\
\text { de } 65 \text { anos. }\end{array}$ \\
\hline 3 & $\begin{array}{l}\text { Augmented reality- } \\
\text {-assisted training } \\
\text { with selected Tai-Chi } \\
\text { movements improves } \\
\text { balance control and } \\
\text { increases lower limb } \\
\text { muscle strength in } \\
\text { older adults: A pros- } \\
\text { pective randomized } \\
\text { trial }\end{array}$ & $\begin{array}{l}\text { Chen et } \\
\text { al. }(2020)\end{array}$ & $\begin{array}{l}\text { RA para aprendizado } \\
\text { de movimentos do } \\
\text { Tai-Chi }\end{array}$ & $\begin{array}{l}\text { Estudo pros- } \\
\text { pectivo rando- } \\
\text { mizado. }\end{array}$ & $\begin{array}{l}28 \text { adultos } \\
\text { de Taiwan } \\
\text { com } 65 \\
\text { anos de } \\
\text { idade e sem } \\
\text { quaisquer } \\
\text { doenças de- } \\
\text { bilitantes. }\end{array}$ \\
\hline
\end{tabular}

Os pesquisadores Calabuig-Moreno et al. (2020) concluem que a RA possui potencial para ser ferramenta de aprendizado, podendo incluir novos movimentos ou melhoria de uma técnica para desenvolver habilidades ofensivas.

Já Chen et al. (2020) faz uma abordagem prática e aplicada da RA para ensinar um sistema de treinamento da técnica Tai-Chi que visa melhorar o desempenho da força e equilíbrio de idosos, prevenindo quedas. A RA foi escolhida por projetar informações virtuais no mundo real, aumentando a consciência e a percepção dos usuários sobre os movimentos adequados. Os autores utilizam o sistema Microsoft Kinect, que detecta os movimentos do corpo por meio de sensor de profundidade e sistema de rastreamento esquelético. $O$ sistema detectava as mudanças críticas de movimento do usuário e as comparava com os movimentos do treinador. 0 resultado da pesquisa demonstra melhoria na força e no equilíbrio dos participantes, embora 
Quadro 3 Artigos da base Ebsco sobre RA aplicada a exercícios físicos de reabilitação.

Fonte Os autores (2021) nem todos os indivíduos mais velhos conseguissem executar os exercícios, chegando a pular ou não executar o programa adequadamente.

Jeon \& Kim (2020) mostram que a partir de um avançado sistema de exercícios para a reabilitação baseado em RA, o UIN-Health, é possível trabalhar a resistência física com práticas aeróbicas e flexibilidade.

Os estudos mostrados no Quadro 3 foram os encontrados na base Ebsco. Essas pesquisas trabalham com reabilitação de pessoas com sequelas que podem ser tratadas com atividades físicas de reabilitação, sendo aplicada, nesse caso, a RA como apoio ao tratamento.

\begin{tabular}{|c|c|c|c|c|c|}
\hline & Artigo & Autores & Foco do Estudo & $\begin{array}{l}\text { Tipo de } \\
\text { Pesquisa }\end{array}$ & Público \\
\hline 1 & $\begin{array}{l}\text { MirrARbilitation: A } \\
\text { clinically-related } \\
\text { gesture recogni- } \\
\text { tion interactive } \\
\text { tool for an AR } \\
\text { rehabilitation } \\
\text { system }\end{array}$ & $\begin{array}{l}\text { Da Gama } \\
\text { et al. } \\
(2016)\end{array}$ & $\begin{array}{l}\text { Avaliar a eficácia do } \\
\text { uso de ferramenta de } \\
\text { reconhecimento de } \\
\text { gestos para o desen- } \\
\text { volvimento de um } \\
\text { sistema de reabilita- } \\
\text { ção de RA interativo }\end{array}$ & $\begin{array}{l}\text { Pesquisa } \\
\text { aplicada. }\end{array}$ & $\begin{array}{l}33 \text { pacientes, } \\
\text { fisioterapeutas e } \\
\text { desenvolvedores } \\
\text { de software. }\end{array}$ \\
\hline 2 & $\begin{array}{l}\text { An Augmented } \\
\text { Reality-Assisted } \\
\text { Therapeutic Heal- } \\
\text { thcare Exercise } \\
\text { System Based on } \\
\text { Bare-Hand Inte- } \\
\text { raction }\end{array}$ & $\begin{array}{l}\text { Zhao, Ong } \\
\text { e Nee } \\
(2016)\end{array}$ & $\begin{array}{l}\text { Apresenta um sis- } \\
\text { tema de exercícios } \\
\text { terapêuticos de } \\
\text { saúde assistidos por } \\
\text { RA de baixo custo e } \\
\text { multimodal para uso } \\
\text { em residências. }\end{array}$ & $\begin{array}{l}\text { Pesquisa } \\
\text { aplicada }\end{array}$ & $\begin{array}{l}\text { Usuários que pre- } \\
\text { cisam aumentar } \\
\text { o movimento dos } \\
\text { dedos, incluindo } \\
\text { jovens saudáveis e } \\
\text { idosos com limita- } \\
\text { da movimentação } \\
\text { dos dedos. }\end{array}$ \\
\hline 3 & $\begin{array}{l}\text { AR-based serious } \\
\text { game framework } \\
\text { for post-stroke } \\
\text { rehabilitation }\end{array}$ & $\begin{array}{l}\text { Hossain et } \\
\text { al., (2016) }\end{array}$ & $\begin{array}{l}\text { Reabilitação de pa- } \\
\text { cientes em reabilita- } \\
\text { ção cognitiva de AVC } \\
\text { por meio de um jogo } \\
\text { com RA. }\end{array}$ & $\begin{array}{l}\text { Pesquisa } \\
\text { de campo } \\
\text { aplicada. }\end{array}$ & $\begin{array}{l}\text { Pacientes sobrevi- } \\
\text { ventes de AVC. }\end{array}$ \\
\hline
\end{tabular}

De acordo com Da Gama et al. (2016) a autovisualização durante a execução da atividade pode melhorar a consciência corporal que resulta em uma execução correta e eficaz, que ainda torna possível ativar a rede visuoespacial adicional do córtex do paciente. Afirmam ainda que a RA é mais eficaz que a RV sobre a precisão do exercício. Esses fatos justificaram a escolha da RA e foram comprovados com o resultado da pesquisa.

A reabilitação testada por Zhao, Ong e Nee (2016) foi para aprimoramento das funções dos dedos das mãos utilizando um sistema simples, composto por duas câmeras de webcam, em que uma ficava posicionada acima da mão e outra ao lado. Com a identificação da luz ideal e o sistema de RA, os resultados foram positivos para os exercícios realizados, sendo o 
sistema considerado divertido, de fácil uso e eficaz para a execução de atividades fisioterápicas.

A pesquisa de Hossain et al. (2016) trabalha com pacientes sobreviventes de AVC, um dos públicos mais estudados em relação à recuperação motora com RA. Os resultados da pesquisa foram positivos quanto ao uso da tecnologia de RA para auxílio à reabilitação, apresentando um comparativo com a realidade virtual que não teve um bom resultado em teste prévio, por causar desconforto nos pacientes.

\section{Discussão}

Os estudos revelaram que a RA tem sido aplicada na vida fitness na forma de jogo e reabilitação. Quanto à gamificação, fundamental no jogo Pokémon Go, verifica-se que pode ser utilizada para envolver pessoas sedentárias, desafiando-as a praticar atividades físicas e se sentirem envolvidas com uso de instrumentos tecnológicos, contribuindo para a redução da ocorrência de hábitos sedentários e, por consequência, das doenças ligadas a eles.

Acerca da reabilitação, verificou-se que a RA está sendo utilizada como ferramenta para direcionar usuários no ensino de atividades físicas, tendo como função principal motivar e orientar corretamente a execução de exercícios físicos, intermediada pelo uso de instrumentos para a visualização das atividades, como câmera, sensores e tela. As evidências apresentadas nos artigos analisados demonstram o aumento efetivo na motivação dos participantes com uso de estratégias de gamificação e feedback imediato.

Quanto ao Pokémon Go, sabe-se que consiste em um jogo baseado em RA. Desse modo, ele foi criado para entreter, e não para orientar usuários na realização de atividades físicas. Marquet, Alberico e Hipp (2018) complementam esta afirmativa explicando que a atividade física não é um objetivo direto do jogo, mas sim um mecanismo pelo qual o jogador pode progredir. Os autores esclarecem que algumas funcionalidades só são desbloqueadas quando certos limites de caminhada são atingidos, e alguns locais-chave no jogo como os pokestops, exigem que o jogador esteja fisicamente próximo para ser usado.

Por se tratar de um videogame ativo, ou seja, que exige que o jogador se movimente, consistiu em um novo fenômeno social que pôde beneficiar a saúde pública. Por esse motivo, de acordo com os artigos coletados nesta revisão, este jogo aumentou a atividade física para os jogadores (ESCARAVAJAL-RODRÍGUEZ, 2018), promovendo: (i) o aumento estatisticamente significativo no tempo gasto caminhando, correndo, andando de bicicleta e patinando (KOGAN ET AL, 2017); (ii) maior frequência em caminhadas e corridas em jogadores sedentários (WONG, 2017) e; (iii) aumento de 1.526 passos diários (MARQUET, ALBERICO E HIPP, 2018), tornando-se uma ferramenta útil para promoção da saúde entre jovens adultos. 
Os estudos analisados também associam outros benefícios ao jogo Pokémon Go, como o maior nível de concentração, atenção seletiva e sociabilidade, quando comparado aos não-jogadores (RUIZ-ARIZA ET AL, 2018), a diminuição da ansiedade em situações como sair de casa, conversar com estranhos e ir a novos lugares (KOGAN ET AL, 2017) e o fomento às relações sociais e descoberta da cidade e da natureza (ESCARAVAJAL-RODRÍGUEZ, 2018).

Porém, apesar dos potenciais benefícios para a saúde obtidos com o Pokémon Go, os estudos de Wong (2017) e Marquet, Alberico e Hipp (2018) apontam que os resultados não oferecem um impacto substancial nos níveis de atividade física dos jogadores, sendo geralmente fracos os ganhos que oferece. Dessa forma, Wong (2017) explica que embora não possa ser utilizado para substituir o exercício normal, uma vez que seus benefícios para a saúde vascular são limitados, sistemas como o jogo Pokémon Go podem ser usados como ponto de partida para pessoas sedentárias iniciarem um estilo de vida ativo.

Importa frisar que existe um desafio similar a todos os jogos, que consiste na diminuição do interesse e nível de entusiasmo, que se dá ao longo do tempo. De acordo com Boulos et al. (2017) a maioria das pessoas ficará entediada com um jogo em algum momento, entre 4 ou 6 meses. Dessa forma, o aumento da prática de exercícios físicos proporcionada pelo Pokémon Go não é "sustentável", como se percebe atualmente pela falta de interesse das pessoas no jogo. Todavia, importa compreender a ligação entre características específicas de jogo e maior atividade física para aprender com a experiência Pokémon Go e aprimorar o design futuro de jogos para a saúde (MARQUET, ALBERICO E HIPP, 2018), além de tê-lo como referência no fornecimento de informações para criação de novas estratégias na promoção da saúde (WONG, 2017).

Quanto à RA aplicada à atividade física para reabilitação, existe um senso comum dos pesquisadores, a de que a tecnologia é ferramenta útil para a motivação da prática de atividades de reabilitação. A pesquisa bibliométrica de Calabuig-Moreno et al. (2020) já destacava em seus achados essa informação, que se confirmou nos resultados de Jeon \& Kim (2020), Chen et al. (2020), Da Gama et al. (2016), Zhao, Ong e Nee (2016) e Hossain et al. (2016).

A RA pode ser utilizada no aprendizado de movimentos ou aprimoramento da sua técnica esportiva, além da melhoria de habilidades como reação, coordenação e habilidades usadas em exercícios de reabilitação, por exemplo (CALABUIG-MORENO et al., 2020). Melhora também a compreensão dos exercícios, devido ao desenvolvimento da consciência corporal pela autovisualização na tela, percepção do erro e o uso de padrões biomecânicos da forma ideal de realizar o exercício (DA GAMA et al., 2016), sendo ainda motivacional para os praticantes.

A RA tem seu potencial motivador por ajudar a manter o foco na atividade realizada, pela autovisualização que permite monitorar se o exercício está corretamente executado, distraindo a percepção da dor du- 
rante os exercícios (DA GAMA et al., 2016). Adiciona-se a isso a gamificação como estrutura de "serious game" associado a feedback tátil e audiovisual (HOSSAIN et al., 2016), o sistema com níveis e pontuação (DA GAMA et al., 2016), e ainda sistemas vestíveis para estimular e encorajar com o feedback tátil (ZHAO, ONG e NEE, 2016). Tais fatores agem como estímulos e motivadores por manter a atenção do praticante enquanto se encontra imerso no sistema.

O feedback imediato é um fator chave para compreensão e correta execução da tarefa ou atividade, seja visual ou audiovisual e tátil. o feedback visual na tela é importante para analisar a usabilidade do equipamento, observando os cuidados com o excesso de informação para não confundir o participante. Por exemplo, a sinalização trabalhada nas áreas da musculatura do corpo, durante um exercício físico, pode não ser facilmente compreendida pelos praticantes (DA GAMA et al., 2016).

Outra vantagem de um sistema baseado em RA é a possibilidade de execução das atividades em casa, reduzindo visitas às clínicas (DA GAMA et al., 2016), além de ser fácil de interagir e compreender (HOSSAIN et al., 2016). Todavia, usuários mais velhos possuem maior dificuldade para executar os exercícios, sendo recomendável atividades personalizadas, de acordo com a capacidade individual de cada um (CHEN et al., 2020). Nesse caso, é desejável contratar um especialista apto a controlar o equipamento para selecionar as atividades mais adequadas (JEON \& KIM, 2020).

Contraditoriamente, apesar do crescente número de estudos que aplicam tecnologias emergentes na Educação Física, profissionais da área desconfiam que a RA pode trazer melhorias na prática dos exercícios, sendo esta uma barreira que precisa ser superada (CALABUIG-MORENO et al., 2020). Calabuig-Moreno et al. (2020) esclarecem que isso ocorre porque os profissionais acreditam que seria uma carga desnecessária de trabalho. Ainda assim, parece ser uma realidade treinar especialistas para usar e controlar aparelhos com RA, principalmente para apoiar idosos (JEON \& KIM, 2020). Essa dificuldade deve ser resolvida com o apoio de profissionais de Educação Física, Fisioterapeutas, Desenvolvedores, Designers, entre outros, que possam desenvolver tecnologias para a prática de exercícios físicos.

\section{Considerações Finais}

A partir da análise realizada por meio desta pesquisa, foi possível obter um panorama da aplicação da RA na vida fitness. Conforme os dados analisados, a RA é investigada em dois grupos de aplicação, a gamificação e as aplicações para reabilitação física. Verifica-se assim que o objetivo da pesquisa de investigar as formas de aplicação da RA para orientar pessoas na realização de atividades físicas, tanto para aumentar a qualidade de vida quanto para diminuir o surgimento de doenças ligadas ao sedentarismo, foi alcançado.

Nos estudos fica clara a capacidade da RA para orientar corretamente a prática de atividades físicas, influenciando positivamente a moti- 
vação para concluir os exercícios pela autovisualização e feedbacks imediatos dados por meio de recursos táteis, visuais e sonoros. Logo, a tecnologia RA tem demonstrado uso potencial para auxiliar a recuperação de pacientes com dificuldades motoras e cognitivas, além de ser usada com êxito na prática e no estímulo de exercícios para prevenção de perda de movimentos e desenvolvimento da musculatura em idosos.

Embora o jogo Pokémon Go tenha estimulado a prática de exercícios físicos pelo mecanismo de progressão do jogador, ele não foi criado para orientar atividades físicas, e sim para entreter. Ainda que esse jogo não tenha oferecido benefícios significativos à saúde e impactos substanciais nos níveis de atividade física dos jogadores, este serviu para constatar que jogos em RA podem estimular pessoas sedentárias a iniciar um novo estilo de vida. Complementarmente, a gamificação intrínseca ao jogo pode ser usada como referência na criação de estratégias para aplicações voltadas à promoção da saúde, como a inclusão de avatares, conquistas e rede social como propostas alinhadas às necessidades de relacionamento e compartilhamento entre usuários. Ademais, pela análise de conexões com outras áreas temáticas, notou-se que Pokémon Go apresentou elevada difusão.

Como primeira constatação deste estudo, tem-se a confirmação da eficácia da RA para aplicações terapêuticas, principalmente quando relacionada à motivação dos usuários e a correta execução dos exercícios, ambas reforçadas por meio da autovisualização. Uma segunda evidência é a compreensão da necessidade de integração da RA com outros dispositivos para dar feedback imediato ao usuário, seja de forma sonora, visual ou tátil. Por fim, constata-se a existência de barreiras à tecnologia: (i) a desconfiança de instrutores de educação física sobre a efetividade do uso da RA como apoio às práticas físicas e; (ii) a dificuldade de pessoas idosas utilizarem as tecnologias emergentes, sendo necessário receberem auxílio de outras pessoas para configurar o equipamento.

O método de revisão, proposto por Tranfield, Denyer e Smart (2003), possui um processo adequado ao protocolo de busca que permite direcionar objetivamente a pesquisa. Ordena e auxilia cada fase da pesquisa, desde a forma de seleção até a análise dos estudos, fornecendo um panorama do estado da arte das publicações relacionadas à temática em questão. Este método proporcionou embasamento teórico e metodológico para nortear o desenvolvimento de projetos futuros em RA para vida fitness. Por ser um método sistematizado, é replicável para a realização de novas buscas.

Notou-se relativo domínio de Estados Unidos e Reino Unido nas publicações em vida fitness com RA aplicada, sendo esta área a mais destacada entre as que configuram entre as publicações elegíveis no estudo. Com as opções de exportação para formato de extensão csv e demais recurso da base Scopus, associado com uso da ferramenta VOSviewer possibilitou ampla oportunidade de diagnósticos e inferências.

A pesquisa apresenta limitações. Verificou-se que a utilização de um conjunto de palavras-chaves semelhantes pode trazer resultados diferentes nas bases de dados utilizadas por esta pesquisa, o que sugere que, 
caso outros termos fossem utilizados, mais estudos seriam incluídos na revisão. Assim, as limitações do trabalho representam oportunidades para trabalhos futuros, sugerindo-se a replicação do método em novas bases de dados, ou ainda, a utilização de outros termos para as palavras-chaves. Sugere-se também, a utilização dos insights e resultados aqui apresentados para desenvolver aplicações para RA voltadas à prática de atividades físicas.

Como contribuições para a área de Tecnologia e Design, esta pesquisa apresentou a vida fitness como uma área promissora para aplicações de RA, sobretudo no contexto de isolamento social, em que a tecnologia vem auxiliar as novas formas de trabalho, lazer e socialização. Verifica-se ainda a possibilidade da aplicação da RA na promoção da saúde, enquanto ferramenta de interação e a motivação do usuário como forma de aprimorar sua experiência e imersão no ambiente real e virtual.

Este estudo estimula a realização de novas pesquisas aplicadas às gamificações que pretendem trazer melhor qualidade de vida e impactos positivos a vida dos usuários. $\mathrm{O}$ estudo contribui ainda para a divulgação e estímulo do uso da RA com foco na experiência do usuário, dado os ganhos que oferece aos indivíduos por abrir novas possibilidades e funcionalidades no campo da saúde e bem-estar.

\section{Agradecimentos}

This research, carried out within the scope of the Samsung-UFAM Project for Education and Research (SUPER), according to Article 48 of Decree $\mathrm{n}^{\circ}$ 6.008/2006 (SUFRAMA), was funded by Samsung Electronics of Amazonia Ltda., under the terms of Federal Law no 8.387/1991, through agreement 001/2020, signed with Federal University of Amazonas and FAEPI, Brazil. 


\section{Referências}

BOULOS, M. N. K. et al. From urban planning and emergency training to Pokémon Go: applications of virtual reality GIS (VRGIS) and augmented reality GIS (ARGIS) in personal, public and environmental health. International Journal of Health Geographics, 2017.

CALABUIG-MORENO, F., GONZÁLEZ-SERRANO, M. H., FOMBONA, J., \& GARCÍA-TASCÓN, M. (2020). The emergence of technology in physical education: A general bibliometric analysis with a focus on virtual and augmented reality. Sustainability, 12(7), 1-23. https://doi.org/10.3390/su12072728

CHEN, P. J., PENN, I. W., WEI, S. H., CHUANG, L. R., \& SUNG, W. H. (2020). Augmented reality-assisted training with selected Tai-Chi movements improves balance control and increases lower limb muscle strength in older adults: A prospective randomized trial. Journal of Exercise Science and Fitness, 18(3), 142-147. https://doi.org/10.1016/j.jesf.2020.05.003

DA GAMA, A. E. F. et al. MirrARbilitation: A clinically-related gesture recognition interactive tool for an $A R$ rehabilitation system. Computer Methods and Programs in Biomedicine, v. 135, p. 105-114, 2016.

ESCARAVAJAL-RodrígueZ, J. C. Pokémon Go and its Influence on Spanish Facebook Users. Apunts. Educación Física y Deportes, Julho-Setembro 2018. 38-49.

HOSSAIN, M. S. et al. AR-based serious game framework for post-stroke rehabilitation. Multimedia Systems, v. 22, n. 6, p. 659-674, 2016.

JEON, S., \& KIM, J. (2020). Effects of augmented-reality-based exercise on muscle parameters, physical performance, and exercise self-efficacy for older adults. International Journal of Environmental Research and Public Health, 17(9). https://doi.org/10.3390/ ijerph17093260

JETTER, JÉRÔME, EIMECKE, J., \& RESE, A. (2018). Augmented reality tools for industrial applications: What are potential key performance indicators and who benefits? Computers in Human Behavior, 87(May), 18-33. https://doi.org/10.1016/j.chb.2018.04.054

KOGAN, L. et al. A pilot investigation of the physical and psychological benefits of playing Pokémon Go for dog owners. Computers in Human Behavior, n. 76, 2017. 431437.

MA, B. D. et al. Pokémon GO and physical activity in Asia: multilevel study. Journal of Medical Internet Research, v. 20, n. 6, p. 1-12, 2018.

MARQUET, O.; ALBERICO, C.; HIPP, A. J. Pokémon Go and physical activity among college students. A study using Ecological Momentary Assessment. Computers in Human Behavior, n. 81, 2018. 215-222.

MILGRAM, P., \& KISHINO, F. (1994). A Taxonomy of Mixed Reality Visual Displays. $12,1-15$.

NG, Y.-L. et al. Effectiveness of virtual and augmented reality-enhanced exercise on physical activity, psychological outcomes, and physical performance: A systematic review and meta-analysis of randomized controlled trials. Computers in Human Behavior, 2019. 278-291. 
RUIZ-ARIZA, A. et al. Effect of augmented reality game Pokémon GO on cognitive performance and emotional intelligence in adolescent young. Computers \& Education, $\mathrm{n}$. 116, 2018. 49-63.

TRANFIELD, D.; DENYER, D.; SMART, P. Towards a Methodology for Developing Evidence-Informed Management Knowledge by Means of Systematic Review. British Journal of Management, 14, 2003. 207-222.

WHO. WHO guidelines on physical activity and sedentary behaviour. World Health organization. Genebra. 2020.

WONG, F. Y. Influence of Pokémon Go on physical activity levels of university players: a cross sectional study. International Journal of Health Geographics, 16, n. 8, 2017.

ZHAO, M. Y.; ONG, S. K.; NEE, A. Y. C. An Augmented Reality-Assisted Therapeutic Healthcare Exercise System Based on Bare-Hand Interaction. International Journal of Human-Computer Interaction, v. 32, n. 9, p. 708-721, 2016. 